\title{
Evaluation of an App to Support Healthy Living by Older Adults
}

\author{
Zaidatol Haslinda, Abdullah Sani \\ Department of Computer Science, \\ University of York, United Kingdom \\ zas508@york.ac.uk
}

\author{
Helen Petrie \\ Department of Computer Science, \\ University of York, United Kingdom \\ helen.petrie@york.ac.uk
}

\begin{abstract}
Smartphone and tablet computers apps are a promising tool to promote healthy attitudes and behaviours among older adults. A tablet computer app to support older adults in maintaining good nutrition and hydration was developed to allow them to track their intake of fruit and vegetables and appropriate liquids. A highly user-centred design lifecycle was used which culminated in a field study with fifteen older adults who used the app for two weeks. Two main themes emerged from the results of their experiences with the app: ease of use and usefulness of the app. Although there were some usability issues, overall participants found the app easy to use, straightforward and designed to do what it was supposed to do. For most of the participants, the app acted as a motivational tool helping them to change their daily diet to eat more fruit and vegetables, and to drink more liquid.
\end{abstract}

\section{healthy eating; healthy liquid intake; older adults; tablet computers; mobile apps}

\section{INTRODUCTION}

Dehydration is a serious health problem, especially for older adults ${ }^{1}$. In the United Kingdom (UK), according to the National Health Services (NHS), dehydration is often associated with other health problem such as malnutrition (NHS, 2015). These health problems are often associated with psychological factors such as bereavement and physiological changes such as dental and oral status that influences liquid and nutrition intake. In addition, factors such as lack of knowledge in consuming the right amount and type of liquid and nutrition intake also contributes to these health problems.

In relation to liquid intake, a four-year survey by Public Health England and the Food Standards Agency found that the average daily non-alcoholic liquid intake of older adults aged 65 and above in the UK was only 1.2 litres for men and 1.3 litres for women (Bates et al., 2014). This is below the recommendations provided by the British Nutrition Foundation (BNF) that men drink 2 litres of nonalcoholic liquids per day, and that women drink 1.6 liters (BNF, 2017).

Poor hydration can lead to tiredness, poor mental performance, physical weakness, dizziness and

${ }^{1}$ We defined older adults as one who is aged 65 years or more increased risk of falls (Masento et al. 2014; Frangeskou et al. 2015). Furthermore, prolonged dehydration can lead to longer hospital admission, increased morbidity and mortality rate (ElSharkawy et al., 2016).

Good nutrition is also a problem for older adults, in particular a lack of fruit and vegetables (FV). The World Health Organization (WHO, 2016) and the $\mathrm{NHS}^{2}$ recommends eating at least five servings of 80 grams of fruit and vegetables a day. However, the European Food Information Council (EUFIC) reported that UK's average FV intake was only 258 grams per day (EUFIC, 2012). In addition, the fouryear survey by Public Health England and the Food Standards Agency found that the older adults aged 65 and above in the UK average FV intake was only 320 grams per day (Bates et al., 2014).

Inadequate consumption of FV increases the risk of coronary heart disease (He et al., 2007), increases mortality rate (Bamia et al., 2007), and lowers quality of life (Anderson et al., 2011, Gopinath et al., 2014) Furthermore, the WHO estimated 5.2 million deaths in 2013 were related to inadequate consumption of $\mathrm{FV}$ (WHO, 2016).

\footnotetext{
${ }^{2}$ http://www.nhs.uk/Livewell/5ADAY/Pages/5ADAYhome.aspx
} 
Currently, mobile technologies, particularly mobile apps, are a promising tool to promote healthy attitudes and behaviours among older adults. Maintaining good health is important for physical and mental performance for everyone, and especially important for older people. To achieve this, mobile apps can be used by older adults to self-monitor their diet (Fukuo et al., 2009, Hakobyan et al., 2016), to engage in physical activity (Fan et al., 2012, King et al., 2016), or to remember to take medications (Dalgaard et al., 2013). Most research has focused on overweight individuals with the aim to help them reduce their weight. However, there have also been studies that aim to raise awareness about their wellbeing by self-monitoring wellness (Doyle et al., 2014).

However, little work has been done in designing and evaluating mobile apps for older people to support them in maintaining good nutrition and intake of liquids, in spite of the fact that they are both significant health issues for this age group (Elia and Russel, 2009).

Our preliminary work with two focus groups of older people showed that they are aware of the importance of good nutrition and keeping hydrated. However, the focus groups showed that the older adults have concerns in drinking sufficient liquid, have a lack of knowledge about the recommended daily amount of liquid to drink, the importance of drinking plain water, and the choice of other appropriate liquids to keep hydrated. The focus groups also showed that although currently only one participant uses a tablet computer, the other participants are also interested in using computers and mobile technologies too.

On the basis of information from the focus groups, guidelines for the development of apps for older adults provided by Silva et al., 2015 and Watkins et al., 2014, as well as other research on the use of touchscreens and mobile devices for older adults (Jin et al., 2007, Kobayashi et al., 2011), we designed an app to support older adults in monitoring liquid intake. A low fidelity prototype of the app was evaluated by a group of experts using the collaborative heuristic evaluation method (Petrie and Buykx, 2010). On the basis of that evaluation, a refined version of the low fidelity prototype was developed and evaluated with 20 older adults. From these evaluations, we have developed the final fully functioning version of the app to support good nutrition and hydration in older adults, the MyHealthyLiving app, allowing users to monitor whether they are eating sufficient FV and drinking sufficient liquid.
This paper reports on a field study of the tablet computer version of the app. The remaining of the paper is as follows: Section 2 describes the Method of the study. In particular, Section 2.2 describes the development of the MyHealthyLiving app. Section 3 describes the Results of the study which include both quantitative and qualitative results. Section 4 reports the discussions and conclusions of the study.

\section{METHOD}

\subsection{Design}

A tablet computer app to support older adults to monitor their FV and liquid intake was evaluated by 15 older adults in a two-week field study. To introduce the app, a one-to-one briefing session was conducted prior the field study. To encourage participant to self-monitor their own diet, no contact was made with them during the field study. Participants used their own tablet computer during the field study.

The features of the app consist of the ability to set liquid measurement preference, add or remove FV and liquid consumed, view congratulation messages upon reaching daily target, view intake progress, and read tips on healthy living. At the end of the two week period, participants were asked to complete a short questionnaire and were interviewed. The questionnaire covered overall acceptance of the app, the ease of use of the app, the usefulness of each feature of the app, and whether each feature raises awareness and motivates the participants to eat more fruit and vegetables and drink more liquid and whether their eating and drinking habits changed during the study (all measured with 7-point Likert items). Further comments on each feature of the app were gathered via open-ended questions. The interview explored further what the participants liked and disliked about the app, what was easy and difficult to use, what changes they would like and any other comments about the app.

\subsection{MyHealthyLiving App}

The web-based application, MyHealthyLiving App (hereafter "the MHL App"), was developed for the iPad tablet computer platform based on previous user requirements work (see Introduction). The app was developed using Web-based technologies (i.e. PHP, CSS, and JavaScript) and MySQL as the database system. The app uses a responsive Web design approach that supports dynamic adaptation of the app interface to a device's characteristics (e.g. screen size and device orientation). 
The app followed Apple's design conventions (Apple Inc., 2017) and guidelines for the development of apps for older adults provided by Silva et al. (2015) and Watkins et al., (2014), as well as other research on the use of touchscreens and mobile devices for older adults (Kobayashi et al., 2011; Jin et al., 2007).

The font size for the text was at least $24 p x$. The target size for the buttons was at least $1.5 \mathrm{~cm}$ (height) $\times 1.5 \mathrm{~cm}$ (width) each (Kobayashi et al., 2011). The gap in between targets was at least $5 \mathrm{~mm}$ (Jin et al., 2007). All text was black on a white background.

The features of the app consist of the ability to set liquid measurement preference, add or remove FV and liquid consumed, view congratulatory messages upon reaching daily target, view intake progress, and read tips on healthy living.

To set liquid measurement preference, a pull-down menu with three options was designed. The options included cup, glass, and pint glass (options suggested by participants in our first evaluation). The user could change the liquid measurement option, if needed, at the "Your Preferred Liquid Measurement" page.

To update FV, buttons to add or remove a whole or $1 / 2$ serving were provided (see Figure 1(a)). The "add" buttons are green in colour, and the "remove" buttons are red. The five hearts represents the recommendation daily servings of $\mathrm{FV}$. When there is no intake for the day, the instruction on how to add the intake is provided and the colours of the hearts are grey and the "remove" buttons are disabled (see Figure 1(a)). To add intake, the user taps on any "add" button and an instant update of their intake progress is shown. The progress is shown in two options: 1) via a text message showing the number of servings and the total percentage of the overall daily target and 2) via the updated pictorial image of the hearts (see Figure 1(b)). Upon reaching the daily target, a congratulatory message is shown (see Figure 1(c)). When there is an intake, the "remove" buttons are then enable (see Figure $1(b-c)$ ).

There are two options to view one's progress. The first option is a coloured chart of the last seven consumptions only. The second is a coloured list showing all consumptions. The traffic light metaphor was used to show progress (i.e. red represents $0 \%$ to $50 \%$ of the daily target, amber represents $51 \%$ to $70 \%$, lighter green represents $71 \%$ to $100 \%$, and darker green represents $101 \%$ or more).
There were two options to read tips about healthy eating. The first option uses the "Read Tips on Healthy Living" page, which consists of buttons that link to external websites (e.g. NHS and BNF) about FV and liquid consumption). The second option uses the "Add My Fruit and Vegetable" page. Each time a user interacts with the app (e.g. adding a FV or liquid consumption), the tips at the bottom of the page change randomly (see Figure $1(\mathrm{a}-\mathrm{c})$ ). The second option was also applied in the progress intake page. However, since there is no interaction apart from scrolling the progress list in this page, only one tip is randomly provided each time the user view this page. The tips were all taken from reliable sources in the UK such as from the NHS and BNF.

To maintain consistency, avoid confusion and reduce mental demand (Silva et al., 2015), the design to add intakes, view progress, and read tips were similar for both $\mathrm{FV}$ and liquid.

\subsection{Materials and Equipment}

A user manual and a post-study questionnaire were developed and presented in large print (16pt). The user manual includes detailed explanations of how to use the app.

The post-study questionnaire includes the following rating items (all $1-7$ Likert items with 1 - most negative, 7 - most positive):

- ease of use to update consumption

- usefulness of different options to update consumptions (e.g. add $1 / 2$ serving or remove 1 serving) and to change liquid measurement option feature)

- each app feature (e.g. view progress, read tips) for usefulness, helpfulness in raising awareness and motivation to eat more FV and to drink more liquids

- if the overall usage of the app helped raise awareness or motivation to eat more FV and to drink more liquid

- if eating and drinking habits changed during the study

Plus open-ended questions to gather further comments on the features of the app and demographic information. Participants also completed the System Usability Scale (SUS) (Brooke, 1996).

In addition, a six-question post-study interview schedule was developed. The questions included what participants like and dislike about the app, what was easy and difficult to use, what changes they would like and what other comments they have about the app. 
During the briefing session, participants were shown the app on an iPad Mini (running iOS 9.3.5).
However, during the field study, participants used their own tablet computer.

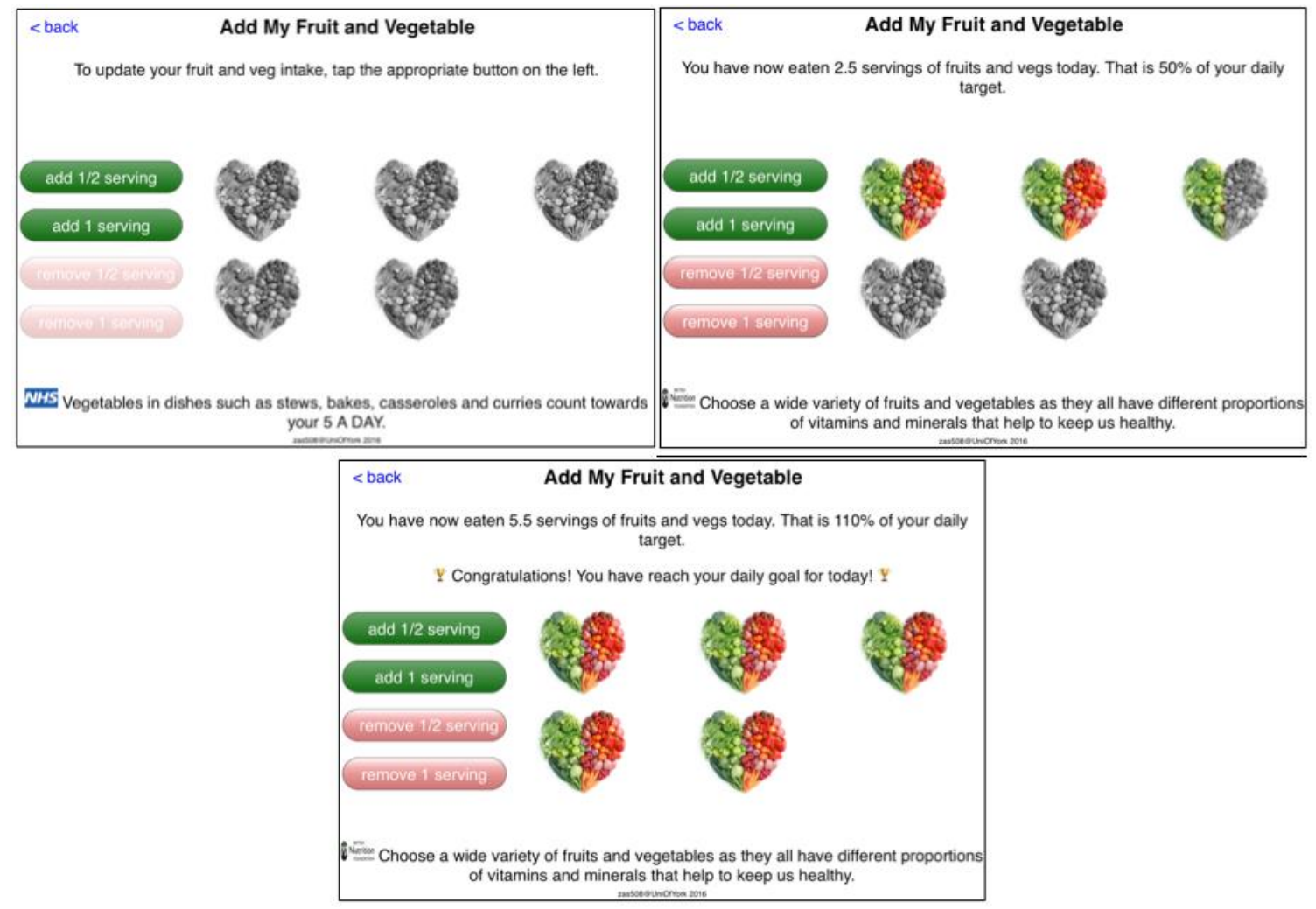

Figure 1: (Clockwise from top left): The add fruit and vegetables page (a) the page with no intake yet, (b) the page with on-going intakes, and (c) the page upon reaching the daily target

\subsection{Participants}

The inclusion criteria were to be 65 years or over, live independently, either alone or with a partner, own a tablet computer, and to have access to the Internet. Fifteen participants took part in the study. Nine participants are women, six are men, their mean age is 70 years with a range from 66 to 78 years. All participants live with a partner. Four participants have secondary school education, four have a bachelors degree, four have a postgraduate degree and three have professional qualifications. Thirteen participants are retirees, two are working part-time. In addition, all participants are Internet users with experience ranging from 3 to 30 years. Eleven participants are computer users with experience ranging from 2 to 30 years. All participants are tablet computer users with experience from 1 month to 6 years. We did not assess the participants' current FV and drinking habits, as this is a sensitive issue.

\subsection{Procedure}

A one-to-one briefing session was held with each participant. The session took place in Interaction $\mathrm{Lab}$ at the Department of Computer Science, a quiet, private location or at the participant's own home. The session included an explanation of the purpose of the study, completing an informed consent form and a demonstration of how to use the app. Participants then explore the app themselves and asked questions. Each session lasted approximately 45 minutes.

After the briefing session, participants were given a unique username, the link of the app, and the user manual. Participants used the app for two weeks on their own, using it as much or as little as they chose. We made no further contact with participants during the two week period. However, participants were welcome to contact the first author if they needed help in using the app.

After the two week period, a debriefing meeting was arranged, again either in Interaction Lab or at the participant's own home. Participants completed a questionnaire about the app and were interviewed by the first author. They were then debriefed about the purpose of the study and invited to ask any questions about it. Participants were offered a gift voucher worth $£ 50$ to thank them for their time and efforts. Each debriefing session lasted approximately 60 minutes.

\subsection{Data analysis}


A Shapiro-Wilk test showed that there was a significant skew in the distribution on majority of the Likert items in the post-study questionnaire. Therefore analysis of the statistical data was done using non-parametric tests. A thematic analysis (Braun and Clarke, 2006) was conducted on the qualitative data to identify the patterns of the participants' experiences of using the app.

\section{RESULTS}

Figure 2 shows the total number of participants adhering to using the app for monitoring their FV and liquid intake during the 14 days of the study. Eight participants used the app for 14 days, 12 participants for 13 days, and 14 participants used for 10 days. Four participants continued using the app after the study completed. Reasons for not entering FV or liquid intake varied, with participants commenting being away from home (e.g. no Internet connection), feeling unwell, attending events during the weekends (e.g. attending weddings, funerals), or forgetting altogether thus missing to record their intakes on a given day.

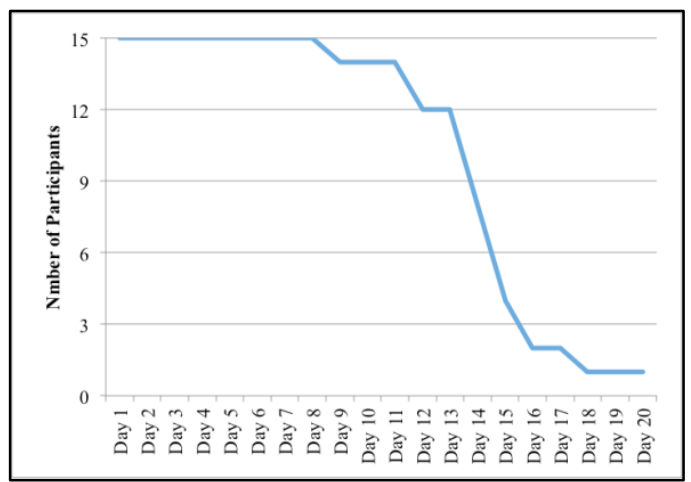

Figure 2: Participants' adherence to using the app

A Spearman's rank-order correlation was run to investigate the relationship between the number of days using the app for FV and mean FV consumptions. There was no significant relationship found between the two measures, $r s=$ .362, $\mathrm{p}=$ n.s. Nor was there a significant relationship between numbers of days using the app for liquid intake and mean liquid consumptions, $r s=.344, p=n . s$. Thus overall the length of using the app does not relate to FV and liquid intakes.

Figure 3 shows the mean ratings of the usefulness of having different options to add or remove a consumption (for example "add $1 / 2$ serving" or "remove 1 glass"). All add features scored above the midpoint of the scale (4). The "remove 1" option for FV and "remove $1 / 2$ " for liquid scored just around the midpoint. All other features scored below the midpoint.

A one-sample Wilcoxon signed-rank test of the usefulness of having different options to update an intake against the neutral mid-point rating of 4 showed that the mean for "add 1" feature for both FV and liquid scores were significant, $Z=120.0, p$ $<0.00(\mathrm{FV})$ and $Z=91.0, p<0.01$ (liquid). There were no other significant effects.

When asked whether the adding $\mathrm{FV} /$ liquid and the option to change liquid measurements features were easy to use, participants gave high ratings. However, a Wilcoxon signed-rank test indicated that the mean of the ease of use of the adding intake features against the neutral mid-point rating of 4 were significant for Add FV $(Z=105.0, p<$ $0.00)$ and Add Liquid $(Z=105.0, p<0.00)$ only.

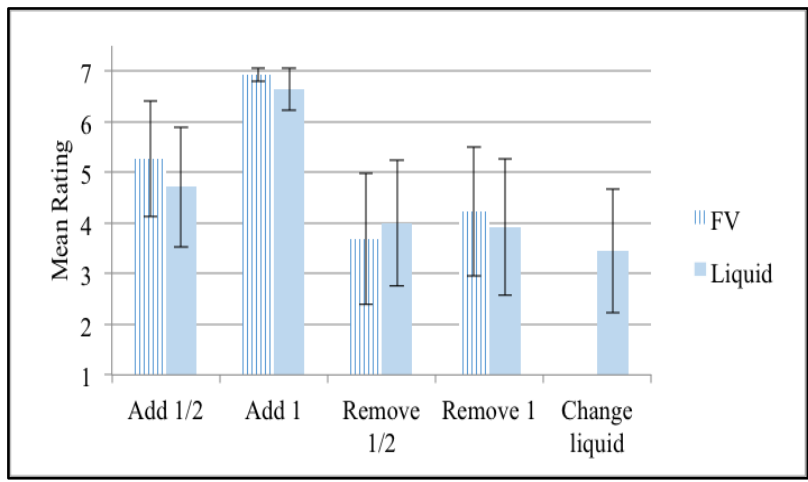

Figure 3: Mean ratings of usefulness for the options to add/remove FV/liquid, and to change the liquid measurement option

Figure 4 shows for the ratings for viewing progress. All the usefulness and awareness ratings were above the midpoint. In term of raising motivation to eat more $\mathrm{FV} /$ drink more liquid, viewing a chart of the last seven consumptions were rated less well than the overall intake view for both FV and liquid.

A Wilcoxon signed-rank test of the usefulness, awareness and motivation of having different options to view progress features against the neutral mid-point rating of 4 showed that the mean of the usefulness of viewing the overall list for both $\mathrm{FV}$ and liquid score was significantly higher than neutral, $Z=74.5, p<0.004(F V)$ and $Z=88.0, p<$ 0.002 (liquid). The mean of the awareness of viewing the overall list for both FV and liquid scores were also significant higher than neutral, $Z=79.0$, $p<0.17$ (FV) and $Z=89.0, p<0.002$ (liquid). There were no other significant effects found. 


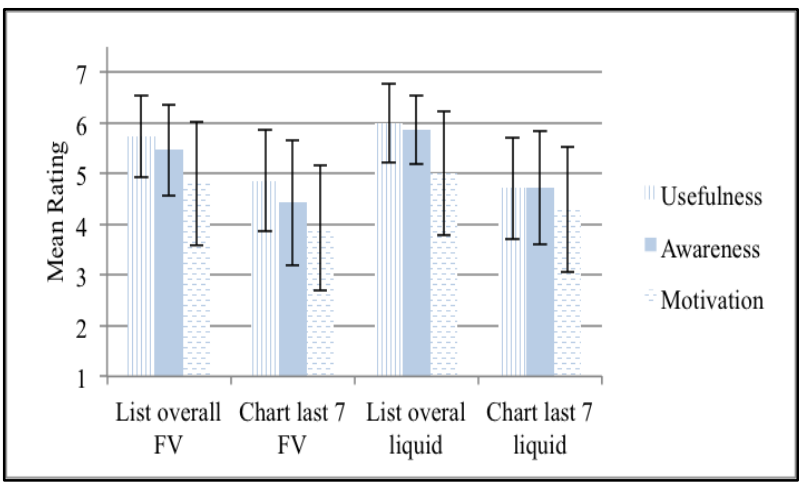

Figure 4: Mean rating of usefulness, awareness, and motivation of having different options to view the progress

Figure 5 shows the rating for the tips options. Reading FV and liquid tips were found useful and raise more motivation to eat more $\mathrm{FV} /$ drink more liquid at the tips page. However, reading FV and liquid tips were found to raise more awareness to eat more FV / drink more liquid while updating consumptions.

A Wilcoxon signed-rank test of the usefulness, awareness and motivation of having different options to read tips against the neutral mid-point rating of 4 showed that only the mean of the usefulness of reading FV and liquid tips at the tips page score were significantly higher than neutral, $Z$ $=83.5, p<0.007$ (FV) and $Z=55.0, p<0.046$ (liquid).

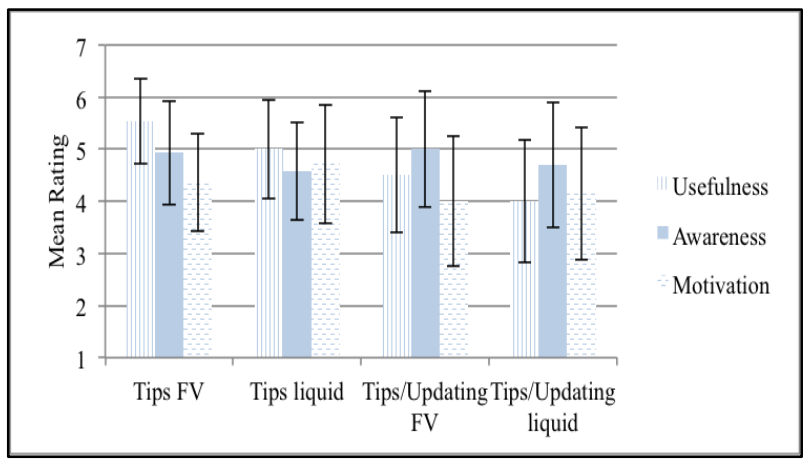

Figure 5: Mean rating of usefulness, awareness, and motivation of having different options to read tips

Figure 6 shows very little difference in terms of usefulness and raising awareness in seeing the congratulations message upon reaching the daily target for both FV and liquid. However, seeing the message motivated to drink more liquid than eating more FV.

A Wilcoxon signed-rank test of the usefulness, awareness and motivation of seeing congratulation messages against the neutral mid-point rating of 4 showed that none of the features were significantly different from neutral.

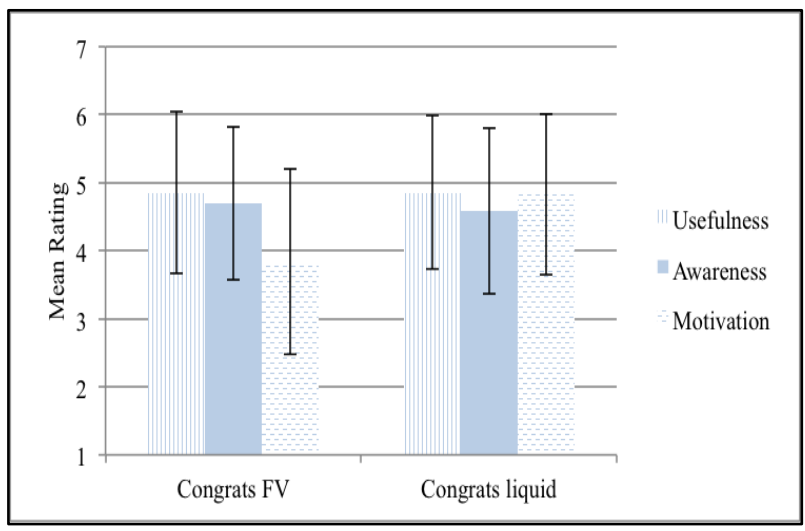

Figure 6: Mean ratings of usefulness, awareness, and motivation of congratulation messages

Figure 7 shows that, overall, using the app made the participants more aware and more motivated to eat more FV and to drink more liquid. Participants moderately agreed with the idea that they ate more FV and drank more liquid during the study than before.

A Wilcoxon signed-rank test of the overall awareness, motivation and eating/drinking habits of using the app against the neutral mid-point rating of 4 showed that only the mean of awareness scores were significantly higher than neutral, $Z=103.0, p$ $<0.001(F V)$ and $Z=103.0, p<0.001$ (liquid).

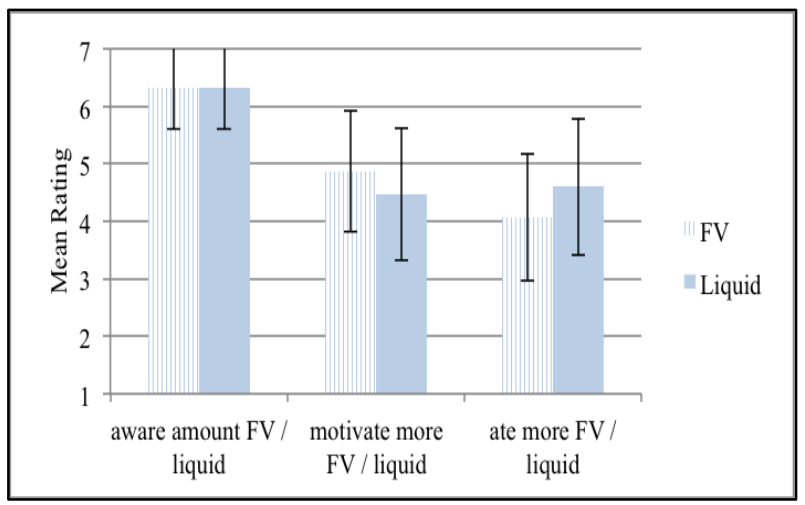

Figure 7: Mean ratings of overall awareness, motivation and eating/drinking habits of using the app

Participants were asked about the most motivating features of the app that encourages them to eat more servings of FV and drink more liquid. For both FV and liquid, the ability to view the list of overall consumption was ranked the highest motivating features by seven participants. The list of overall consumption for both FV and liquid was also found to be more useful, raise more awareness, and motivate them to eat more FV and to drink more 
liquid as compared to viewing the chart of the last seven intakes (see Figure 4, above).

The System Usability Scale (SUS) was used to measure the app's usability (Brooke, 1996). The mean SUS score was 89.17, indicating that the participants found the app highly usable.

\subsection{Analysis of interview questions}

The analysis of what the participants said they liked about the app produced two main themes: (a) ease of use, and (b) usefulness of the app. The findings of each theme are elaborated below.

\subsubsection{Ease of use of the app}

Participants' main comments in terms of the app's usability and acceptability was the ease of use of the app and that it was easy to understand:

that it was easy to use well it was obviously what it was for very straightforward and it was a nice way to record information and I can just press a button and see all my history and that's what I like P10

A majority of participants found the practice session prior to the study was helpful to gain understanding of using the app. In addition, some participants mentioned referring to the user manual prior the study to gain understanding of using the app:

I found it all easy I am not a technical person but once it was explained to me the practice session helped because if I was given to it just like that I don't think I would be confident enough to use it P14

I did read the instruction to begin with that was straightforward so yes, it was very straightforward with both liquid and fruit and vegetables $\mathrm{P} 13$

Further comments from the participants highlighted that the ease to use and the way the app was designed encouraged them to want to use the app. The simplicity of the app was crucial to gain the participants' enthusiasm to use the app:

it is easy to use and importantly the way it is designed makes you want to use it P2

Participants were not fearful of using new technologies, despite having different technology backgrounds, particularly in using a touchscreen. Our findings also suggest that these older adults are interested in investing time and effort in using new technologies as long as the technologies brings benefits for them:
I prefer to play with the system to lose my fear of the system it took me three days to get the understanding of how the app works P5

if it got into taking more of my time l'd get impatient with it I like that I can do it quickly and forget about it and the fact that you can do it anytime P15

\subsubsection{Usefulness of the app}

The analysis revealed four sub-themes in related to the usefulness of the app: (a) raises awareness to eat and drink healthy and to facilitate selfmonitoring diet, (b) encourages positive behaviour change, (c) potential to support memory and enhance learning and (d) potential to improve social interaction. The findings of each sub-theme are elaborated below.

\subsubsection{Raises awareness to eat and drink healthy and to self-monitor diet}

The results revealed that one of the main advantages of using the app is its capability to raise awareness to eat more FV and drink more liquid. This is important for the participants to improve health, longevity, and independence as studies have shown that older adults prefer to be independent for as long as they can (Demiris et al., 2004, Chaudhuri et al., 2015), rather than relying on others (e.g. family members or carers) to perform daily activities. Our participants indicated that the app showed them their current eating and drinking habits, which triggered awareness to eat and drink more healthily;

this study tells me more about myself I did not know that I don't drink enough or eat enough but now be able to see it P15

The app was found useful to keep track of the participants' eating and drinking habits. Participants highlighted the app acted as a motivational tool to support them to self-monitor their diet:

I like the fact that it made you very aware of what you're eating and drinking I find it very helpful because I know you know that we have to eat FV and all but keeping a record of it you know you can see what how much you eat actually it is very helpful P13

Participants felt the app made them reflect on what they have been consuming. Participants also find it interesting to be able to monitor and analyse their intake:

it was a simple way to look back at what I have been eating and drinking in a day quite nice to see quite precisely $\mathrm{P} 11$ 
Each feature of the app was found to positively encourage the participants to self-monitor their diet. Participants emphasized that the bar chart to show their weekly progress was useful to monitor their progress:

I like the bar chart it's good to see it affects my behaviour to get better readings P5

Nevertheless, participants also found the list to show the overall progress interesting to selfmonitor their diet:

I found it [list chart] interesting to follow my whole progress $\mathrm{P} 3$

The use of the traffic light metaphor as a form of feedback was also found to motivate the participant to change their diet and to keep using the app. This finding contradicts with the findings by previous studies, for example by Doyle et al. (2014) and Grimes et al. (2010), where some older adults in their studies did not understand the concept of the traffic light metaphor as a form of feedback while using apps. Our participants commented:

I like the traffic colour code it made me wanting to use the app more so that I can avoid seeing red $\mathrm{P} 5$

it's a motivation to see the 100 per cent and the greens all the time P8

The recommendation of daily servings for $\mathrm{FV}$ and liquid intakes was also found to raise awareness to eat more FV and drink more liquid. This supports findings from previous studies that older adults are more likely to agree using self-monitoring technologies and to adhere to the recommendations when the use of technologies can improve their health (Fowles et al., 2004):

I can see that I am eating and drinking enough according to the recommendation it's good to know the target though I know at sometimes I'm above it and sometimes I am below makes me realise that I have to eat and drink more some of these things $\mathrm{P} 11$

The tips section was also found to raise awareness to eat and drink healthy. The findings show that the tips, both at the tips page and the add intake page, motivated the participants to improve their diet:

this [tips at tips page] is ok because I was conscious of not drinking enough it did motivate me to drink more P14

interesting to see the tips [tips at add intake page] changes all the time keeps me motivated p15
The design, colours and images used in the app also raised awareness to eat and drink healthy. Participants emphasized the simplicity of the interaction design to update an intake was satisfying and the design reminded them to eat and drink healthily:

the hearts the shape the colours it reminds you that this is good for your heart and eating healthy P9

to see the hearts it's more satisfying I enter an intake and this little heart changes colour I like that actually I really really like that P10

\subsubsection{Encourage positive behaviour change}

Apart from raising awareness and encouraging self-monitoring of diet, the app was also found to motivate the participants to make positive changes to their diet:

I think it came on the right time for me there's been quite a lot about drinking water I have decided to cut down a lot on alcohol because it interferes with my sleep I now drink coffee in the morning and then tea later I drink more juices now P5

Participants emphasized the progress options showed clearly how much FV and liquid they consumed, thus motivating them to change their diet. The willingness to change is important for their health as this age group are often linked to various health problem, such as dehydration, that can cause various negative consequences including increased risk of infections of many kinds, increased falls due to dizziness, and difficulties metabolizing medications (Abdallah et al., 2009). Some of the participants commented:

looking at the reds in the graphs does affect me it did made me wanting to drink more and eat more it brings the good effect to change $\mathrm{P} 13$

The congratulations message was also found to be helpful in motivating the participants to eat healthily. Participants were also keen to maintain eating healthily to be able to receive the message:

this message is very encouraging and therefore motivates me to maintain or do better P2

The tips encouraged participants to eat and drink healthily, especially to incorporate more variety of FV and drinks to their diet:

I like the random messages it helps to think about what to eat drink change or buy to fulfil the target P1 
Participants were also surprised to learn how much FV and liquid they consumed on a daily basis and throughout the study:

\section{I was surprised at how much I did drink P7}

Over the period of the trial I increased my daily consumption of fresh fruit and enjoyed eating it P2

In overall, the qualitative findings suggest that the app was successful in promoting positive behaviour change through the use of the various features in the app. The app did successfully raise the participants' awareness to eat and drink healthily, encourage them to self-monitor their diet and it also improved their healthcare knowledge.

\subsubsection{Potential to support memory and enhance learning}

The app was also found to potentially support participants' memory. We did not assess the impact of using the app on participants' memory, however, repeated comments from participants commented on how the app support their memory, an area which this age group often require assistances (Maciuszek et al., 2005). The use of mobile technology has also shown to improve cognitive abilities among older adults (Chan et al., 2016). In our study, participants were required to remember what they had consumed and update their intake and that this could possibly support their memory ability. Comments from participants included:

the app is good at one point because we have to calculate first what we drunk say for example a bottle is $500 \mathrm{ml}$ so it's 12 and half P14

The app was also found to educate the participants with new knowledge and encourage learning. Participants commented that the tips sections provided a better understanding of FV and liquid intake making them want to learn more about healthy living:

I always read the tips to get an idea what is a serving and then update my intake P8

Although some of the participants were not Apple users, they managed to quickly understand how to navigate to using the app. As one of the participants noted:

I realised that the back and logout was all on the left where as most system on the computers they have the $X$ on the right don't they ... but the good thing about your design is that everything is on your left so back back back then you're off $\mathrm{P} 12$

\subsubsection{Potential to support social interaction}

Another less frequently mentioned but important finding is the app can potentially support social interaction among family members, while they improve their dietary habits. We did not explicitly assess the impact of using the app on participants' social interaction, however, the comments indicate that some participants found the app contributed to their social interaction. Two couples who participated in the study used the app like a game, seeing who reached their daily target first. Participants were found sharing their progress with their partners and, one participant shared and analysed his progress with his wife every day, even though she was not part of the study. One of the couple participants further suggested making the app accessible among family members. Comments from participants included:

I like comparing my hearts and cups with [P11] I usually get more perhaps can add social groups among family not with everyone P10

the fact that we used it daily I like that it's an everyday thing each morning I'll enter what I had the previous day and analyse my intakes with my wife P5

These participants made changes to their diet to fulfil the recommended daily target. These examples shows that communicating and social interaction is important (Grimes et al., 2010), be it to analyse daily intake or to compare each other intake's progress, as loneliness, social isolation, and poor diet can cause health problems and often appear as an issue for this age group (Ong et al., 2015).

When asked about the dislikes of the app, participants found the tips section was too general for people of their age, and wanted more specific tips on healthy living. Participants also found the login process ${ }^{3}$ cumbersome since they were using their own tablet computers, especially the fact that the app auto-logged them off if there were no interaction made after a few minutes. Any interaction done after the auto logged out would not be updated in the app. As one of the participant commented:

sometimes when you are log in and updating your liquid in and you want to update your vegetables in it won't let you so you have to

\footnotetext{
${ }^{3} \mathrm{~A} \log$ in was necessary as the participants' data needed to be stored securely on a xxx server.
} 
log out and come back in and check so I found that very fiddly P10

Some participants thought their intakes were updated but only realized that the intakes were not updated once they looked at their progress option, but they could not edit their intake. This contributed to another dislike about the app, that it did not allow editing of previous intakes, especially for days on which participants missed updating their intake. However, some participants raised concerns about changing this feature. They noted that people would perhaps tend to not update their intakes on a daily basis (knowing that they could make updates on later days), which would lead to not using the app accurately. One participant further commented that allowing people to edit previous intakes opens up the possibility of cheating on reporting. Two other participants suggested allowing editing for just a few days back, because people will tend to forget what they had consume if it was far back anyway.

There were suggestions for improving the usability of the app. One participant commented that updating FV using ' $1 / 2$ serving' and ' 1 serving' can be difficult if one does not know what counts as a serving. She suggested having buttons labelled with the serving sizes of different FV, and then one could tap on those buttons to add servings. Three other participants suggested separating the FV and liquid into different options (e.g. apple, broccoli, coffee, juice) so they can see exactly what they have consumed.

Another suggestion was to have more colours representing the $\mathrm{FV}$ consumption rather than only having green and red (see Figure 1(c)). This participant suggested having a panel of colours to appear after tapping the ' $1 / 2$ serving' or ' 1 serving' buttons, and then one could tap on any colours that matches the FV to add servings. There were also participants who preferred having different measurement options to update the liquid intake, for example using mugs or bottles. One participant preferred to be able to enter his own liquid intake volume rather than having a fixed volume labelled on a button.

Two participants suggested having time features when updating an intake. These participants commented that a time feature would help them to remember which update was for which intake. Three participants suggested having bigger gaps between the buttons for adding servings. Four participants commented to use different colours in the app. A number of participants highlighted the tips at the adding intake page was too far from the buttons, and therefore not very noticeable. Two participants suggested adding animation to attract them to read these tips. Three participants noted their main concern at the adding intake page was to add their intake and not to read tips; they wanted to read tips at the tips page.

Two participants suggested showing the exact number of icons for the hearts and cups that correspond to the goal, especially once they have reached their daily goal. One participant noted it was difficult to see whether the intake was updated or not by relying just on the percentage. The other participant noted that it is more satisfying seeing the icon to change colour than seeing the percentage increasing.

There was also some confusion on the date format used in the app, as it followed the United States (US) style. Participants preferred the date to be in the UK format. A number of participants who were not used to the Apple design conventions (Apple, 2017), and were confused to have the "back" button appear on the top-left side of the screen.

\section{DISCUSSION AND CONCLUSIONS}

This paper reports the result of a two week field study to investigate the use of a tablet computer app to support healthy living among older adults, in terms of eating sufficient fruit and vegetables and drinking sufficient liquid.

Participation in the field study was good. Just over half the participants used the app for the whole 14 days, and nearly half used the app for 10 out of the 14 days, and there were no drop-outs. There were a number of legitimate reasons for missing days, but in general participants were eager to use the app.

We included a number of features in the app to raise awareness about healthy eating and drinking and to motivate people to eat more fruit and vegetables and to drink more liquid. All features scored above the midpoint of the rating scale for usefulness except for the removing intake options and the change liquid measurement options. All features also scored above the midpoint of the rating scale for ability to raise awareness and motivation to eat and drink more except for the bar chart for viewing weekly progress, which did not found to be motivating.

Participants also ranked viewing the list of the overall progress for both FV and liquid intakes as the most motivating features that encourage them to eat more FV and to drink more liquid. The list option was also found to be more useful, to raise more awareness, and motivate them to eat more 
FV and to drink more liquid as compared to viewing the chart of the last seven intakes. Arsand et al. (2010) to self-monitor diet for diabetes participants (mean age $=56$ years) also found that users preferred the ability to track and view their entries as compared to other features such as setting personal goals. A review paper on behaviour change techniques of 64 apps on iTunes and Google Play also confirmed that providing feedback on performance is the most frequently used technique to promote health behaviour change (Middelweerd et al., 2014).

The qualitative findings from this study also confirm outcomes of previous studies. Two major themes in related to the participants' experiences of the app were identified. The themes were ease of use and usefulness of the app.

In terms of ease of use, participants found the app easy to use, straightforward and designed to do what it supposed to do. In terms of usefulness, four sub-themes were found related to the participants' experiences of using the app. The sub-themes were (a) raising awareness to eat and drink healthily and to facilitate self-monitoring diet, (b) encouraging positive behaviour change, (c) potential to support memory and enhance learning and (d) potential to improve social interaction.

The two major themes were also found in studies of apps with older adults to improve health by Hakobyan et al. (2016), King et al.,(2013), and Arsan et al. (2010). The current results also support previous findings that older adults find using apps useful as a motivational tool to support maintaining and self monitoring their lifestyle despite having different technology backgrounds, particularly in using touchscreens.

Each feature of the app in the current study was found useful, raised awareness and motivated participants to eat more $\mathrm{FV}$ and to drink more liquid. As noted, this is important for the participants to improve health, longevity, and independence as studies have shown that older adults prefer to be independent for as long as they can (Demiris et al., 2004, Chaudhuri et al., 2015). Furthermore, although not formally tested, the unexpected but encouraging findings from the qualitative findings showed that the app also supported memory abilities, enhanced learning and potentially improved social interaction, areas that are often problematic for older adults (Maciuszek et al., 2005, Ong et al., 2015).

We will take in consideration all comments and suggestions given by our participants in further developing the app, although we are aware that designing an app that fits all users, especially older adults, is perhaps impossible. Future work will also include investigating the suggestions given by our participants to see if it can raise awareness and motivates them to eat more FV and drink more liquid.

One limitation of this study is that participants only used the app for two weeks. This is not a long period of time to establish whether the app would be used if participants had it available for a long period and whether it would continue to motivate them in eating and drinking healthily. That said, health behaviour change is only beneficial when observed over a long period of time (Connor and Norman, 2005). However, the purpose of this study was to establish the usability and overall acceptance of an app in the nutrition and hydration area by older adults, and the results suggest this is very much the case.

\section{ACKNOWLEDGEMENTS}

We would like to thank all our participants for their time and effort to take part in the study. The first author would also like to acknowledge her sponsor, Majlis Amanah Rakyat, Malaysia. We would also like to thank our reviewers for their comments.

\section{REFERENCES}

Abdallah, L., Remington, R., Houde, S., Zhan, L., and Melillo, K.D. (2009) Dehydration reduction in community-dwelling older adults: perspectives of community health care providers. Research in gerontological nursing, 2, (1), 49-57.

Apple Inc. (2017) iOS Human Interface Guidelines. Available at: https://developer.apple.com /library /ios/documentation/userexperience/conceptual/ mobilehig/

Anderson, A. L., Harris, T. B., Tylavsky, F. A., Perry, S. E., Houston, D. K., Hue, T. F., Strotmeyer, E. S. \& Sahyoun, N. R. (2011) Dietary patterns and survival of older adults. Journal of the American Dietetic Association, $111,84-91$

Årsand, E., Tatara, N., Ostengen, G. \& Hartvigsen, G. (2010) mobile phone-based self-management tools for type 2 diabetes: The few touch application. Journal of diabetes science and technology, 4, 328-336.

Bamia, C., Trichopoulos, D., Ferrari, P., Overvad, K., Bjerregaard, L., Tjønneland, A., Halkjaer, J., Clavel-Chapelon, F., Kesse, E. \& BoutronRuault, M.-C. (2007) Dietary patterns and 
survival of older Europeans: the EPIC-elderly study (European Prospective Investigation into Cancer and Nutrition). Public health nutrition, 10, 590-598.

Bates, B., Lennox, A., Prentice, A., Bates, C., Page, P., Nicholson, S., and Swan, G. (2014) National Diet and Nutrition Survey Results from Years 1, 2, 3 and 4 (combined) of the Rolling Programme (2008/2009-2011/2012): A Survey Carried Out on Behalf of Public Health England and the Food Standards Agency. Available at: https://www.gov.uk/government/uploads/system/u ploads/attachment_data/file/594360/NDNS_Y1_to_ 4_UK_report_executive_summary_revised_February 2017.pdf

British Nutrition Foundation (2017) Healthy Hydration Guide. Available at: https://www.nutrition.org.uk/healthyliving/hydratio n/healthy-hydration-guide.html

Braun, V., and Clarke, V. (2006) Using thematic analysis in psychology. Qualitative research in psychology, 3, (2), pp. 77-101

Brooke, J. (1996) SUS - A quick and dirty usability scale. Usability Evaluation in Industry. pp. 4-7. Taylor \& Francis Ltd, London

Chan, M.Y., Haber, S., Drew, L.M., and Park, D.C. (2016) Training Older Adults to Use Tablet Computers: Does It Enhance Cognitive Function?. The Gerontologist, 56, (3), pp. 475484

Chaudhuri, S., Kneale, L., Le, T., Phelan, E., Rosenberg, D., Thompson, H., and Demiris, G. (2016) Older Adults' Perceptions of Fall Detection Devices. Journal of Applied Gerontology, $\quad 0, \quad(0), \quad$ doi: https://doi.org/10.1177/0733464815591211

Conner, M., and Norman, P. (2005) Predicting health behaviour, McGraw-Hill Education, UK

Conover, W.J., and Iman, R.L. (1981) Rank transformations as a bridge between parametric and nonparametric statistics. The American Statistician, 35, (3), pp. 124-129

Dalgaard, L.G., Gronvall, E., and Verdezoto, N. (2013) MediFrame: A Tablet Application to Plan, Inform, Remind and Sustain Older Adults' Medication Intake. In: IEEE International Conference on Healthcare Informatics (ICHI), USA, 9-11 September, pp. 36-45, IEEE Conference Publications

Demiris, G., Rantz, M.J., Aud, M.A., Marek, K.D., Tyrer, H.W., Skubic, M., and Hussam, A.A.
(2004) Older adults' attitudes towards and perceptions of 'smart home' technologies: a pilot study. Informatics for Health and Social Care, 29, (2), pp. 87-94

Doyle, J., Walsh, L., Sassu, A., and McDonagh, T. (2014) Designing a Wellness Self-Management Tool for Older Adults-Results from a Field Trial of YourWellness. In: 8th International Conference on Pervasive Computing Technologies for Healthcare, Germany, 20-24 May, pp. 134-141, ICST

Elia, M., and Russell, C. (2009) Combating malnutrition: recommendations for action. Available at: http://www.bapen.org.uk/pdfs/reports/advisory_g roup_report.pdf

El-Sharkawy, A.M., Virdee, A., Wahab, A., Humes, D.J., Sahota, O., Devonald, M.A.J., and Lobo, D.N. (2016) Dehydration and clinical outcome in hospitalised older adults: A cohort study, European Geriatric Medicine, doi: http://dx.doi.org/10.1016/j.eurger.2016.11.007

EUFIC (2012) Fruit and vegetable consumption in Europe - do Europeans get enough? Available at: http://www.eufic.org/article/en/expid/Fruitvegetable-consumption-Europe/

Fan, C., Forlizzi, J., and Dey, A. (2012) Considerations for technology that support physical activity by older adults. In: Proceedings of the 14th International ACM SIGACCESS Conference on Computers and Accessibility, USA, 22-24 October, pp. 33-40, ACM, New York

Frangeskou, M., Lopez-Valcarcel, B., and SerraMajem, L. (2015) Dehydration in the elderly: A review focused on economic burden. The Journal of Nutrition, Health \& Aging, 19, (6), pp. 619-627

Fowles, J.B., Kind, A.C., Craft, C., Kind, E.A., Mandel, J.L., and Adlis, S. (2004) Patients' interest in reading their medical record: relation with clinical and sociodemographic characteristics and patients' approach to health care. Archives of Internal Medicine, 164, (7), pp. 793-800

Fukuo, W., Yoshiuchi, K., Ohashi, K., Togashi, H., Sekine, R., Kikuchi, H., Sakamoto, N., Inada, S., Sato, F., and Kadowaki, T. (2009) Development of a Hand-Held Personal Digital AssistantBased Food Diary with Food Photographs for Japanese Subjects. Journal of the American Dietetic Association,109,(7), pp. 1232-1236 
Gopinath, B., Russell, J., Flood, V. M., Burlutsky, G. \& Mitchell, P. (2014) Adherence to dietary guidelines positively affects quality of life and functional status of older adults. Journal of the Academy of Nutrition and Dietetics, 114, 220229.

Grimes, A., Kantroo, V., and Grinter, R.E. (2010) Let's play!: mobile health games for adults. In: Proceedings of the 12th ACM international conference on Ubiquitous computing, Denmark, 26-29 September, pp. 241-250, ACM, New York

Hakobyan, L., Lumsden, J., Shaw, R. \& O'sullivan, D. (2016) A longitudinal evaluation of the acceptability and impact of a diet diary app for older adults with age-related macular degeneration. Proceedings of the 18th International conference on human-computer interaction with mobile devices and services. Italy, 6-9 September, pp. 124-134, ACM, New York

He, F., Nowson, C., Lucas, M. \& Macgregor, G. (2007) Increased consumption of fruit and vegetables is related to a reduced risk of coronary heart disease: meta-analysis of cohort studies. Journal of human hypertension, 21, 717-728.

Jin, Z. X., Plocher, T. \& Kiff, L. (2007) Touch screen user interfaces for older adults: button size and spacing. International Conference on Universal Access in Human-Computer Interaction. Springer, 933-941.

King, A. C., Hekler, E. B., Grieco, I. A., Winter, S. J., Sheats, J. L., Buman, M. P., Banerjee, B., Robinson, T. N. \& Cirimele, J. (2013) Harnessing different motivational frames via mobile phones to promote daily physical activity and reduce sedentary behavior in aging adults. Plos one, 8, e62613.

King, A.C., Ahn, D.K., Oliveira, B.M., Atienza, A.A., Castro, C.M., and Gardner, C.D. (2008) Promoting physical activity through hand-held computer technology. American journal of preventive medicine, 34, (2), pp. 138-142

Kobayashi, M., Hiyama, A., Miura, T., Asakawa, C., Hirose, M. \& Ifukube, T. (2011) Elderly user evaluation of mobile touchscreen interactions. Human-Computer Interaction-INTERACT 2011. Springer.

Lewis, C. (1982) Using the "thinking-aloud" method in cognitive interface design. IBM TJ Watson Research Center, New York
Lloyd-Williams, M., Kennedy, V., Sixsmith, A., and Sixsmith, J. (2007) The end of life: a qualitative study of the perceptions of people over the age of 80 on issues surrounding death and dying. Journal of pain and symptom management, 34 , (1), pp. $60-66$

Maciuszek, D., Aberg, J., and Shahmehri, N. (2005) What help do older people need?: constructing a functional design space of electronic assistive technology applications. Proceedings of the 7th international ACM SIGACCESS Conference on Computers and Accessibility, USA, 9-12 October, pp. 4-11, ACM, New York

Masento, N.A., Golightly, M., Field, D.T., Butler, L.T., and van Reekum, C.M. (2014) Effects of hydration status on cognitive performance and mood. British Journal of Nutrition, 111, (10), pp. 1841-1852

Middelweerd, A., Mollee, J. S., Van Der Wal, C. N., Brug, J., and Te Velde, S. J., (2014) Apps to promote physical activity among adults: a review and content analysis. International journal of behavioral nutrition and physical activity, vol. 11, no. 1, pp. 97

NHS (2015) Guidance-Commissioning Excellent Nutrition and Hydration 2015-2018. Available at: $\quad$ https://www.england.nhs.uk/wpcontent/uploads/2015/10/nut-hyd-guid.pdf

Ong, A.D., Uchino, B.N., and Wethington, E. (2015) Loneliness and Health in Older Adults: A MiniReview and Synthesis. Gerontology, 62, (4), pp. 443-449

Petrie, H., and Buykx, L. (2010) Collaborative Heuristic Evaluation: improving the effectiveness of heuristic evaluation. Proceedings of UPA 2010 International Conference, Germany, 24-28 May Omnipress. Available at: http://upa.omnibooksonline.com/index.htm2010

Silva, P.A., Holden, K., and Jordan, P. (2015) Towards a List of Heuristics to Evaluate Smartphone Apps Targeted at Older Adults: A Study with Apps that Aim at Promoting Health and Well-being. In: Proceeding of the 48th Annual Hawaii International Conference on System Sciences, USA, 5-8 January, pp. 32373246, CPS, California

Watkins, I., Kules, B., Yuan, X. \& Xie, B. 2014. Heuristic Evaluation of Healthy Eating Apps for Older Adults. Journal of Consumer Health On the Internet, 18, 105-127. 
WHO (2016) Increasing fruit and vegetable consumption to reduce the risk of noncommunicable diseases. Available at: http://www.who.int/elena/titles/fruit_vegetables_ ncds/en/ 